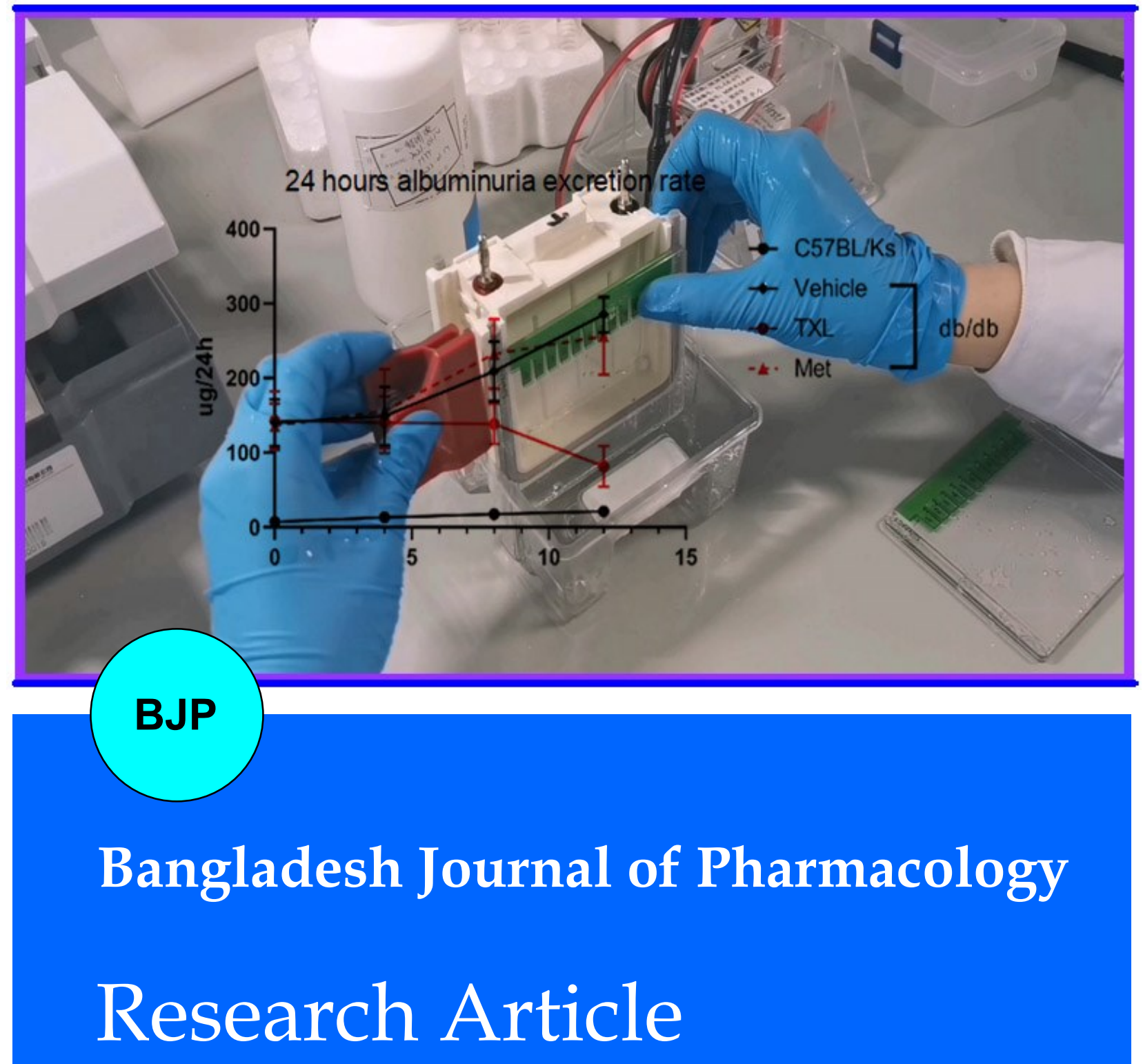

Tongxinluo preserves the renal function in diabetic kidney disease via protecting the HK-2 cells from the patterns of programmed cell death 


\title{
Tongxinluo preserves the renal function in diabetic kidney disease via protecting the HK-2 cells from the patterns of programmed cell death
}

\author{
Shuang Shen', Yunlong Hou ${ }^{2}$ and Yiling $\mathrm{Wu}^{1}$
}

${ }^{1}$ Nanjing University of Chinese Medicine, Nanjing 210046, China; ${ }^{2}$ Key Disciplines of State Administration of Traditional Chinese Medicine for Collateral Disease, Shijiazhuang 050011, China.

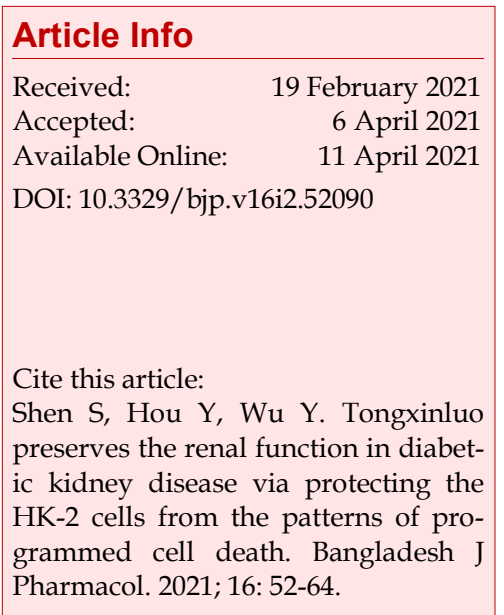

\begin{abstract}
This study was explored the renoprotective effect of Tongxinluo (a Traditional Chinese Medicine) on diabetic kidney disease and its underlying mechanism. The results revealed significant $(\mathrm{p}<0.05)$ up-regulation of the expression of Bcl-2 and down-regulation of NF-kB p-p65, ASC, NLRP3, caspase-1, cleaved GSDMD, IL-1 $\beta$, caspase-3, Bax and the ratio of Bcl-2 and Bax in immortalized proximal tubular HK-2 cells cultured by hyperglycemia combined with hyperlipidemia. In $\mathrm{db} / \mathrm{db}$ mice, Tongxinluo treatment substan -tially decreased the 24 hours albuminuria excretion rate and the urine albumin-creatinine ratios and renal morphologic abnormalities. Similarly, the levels of NF-кB p-p65, NLRP3, caspase-1, cleaved GSDMD, IL-1 $\beta$, caspase-3, and the ratio of Bcl-2 and Bax were down-regulated by Tongxinluo treatment in the kidney samples of $\mathrm{db} / \mathrm{db}$ mice $(\mathrm{p}<0.05)$. Taken together, according to the anti-pyroptosis and anti-apoptosis effect of Tongxinluo, it shows the potential to be effective for the treatment of diabetic kidney disease.
\end{abstract}

\section{Introduction}

Diabetic kidney disease is a leading cause of advanced kidney disease and a typical complication of diabetes (Anders et al., 2018). Although the development of clinical therapy and the pathogenesis for diabetic kidney disease have made significant progress, the progression of it still cannot be controlled. Therefore, the discovery of a novel and effective treatment for diabetic kidney disease patients is required.

Typically, the diabetic kidney disease is a progressive microvascular complication arising from diabetes that is mainly concentrated on the glomerulus. Tubulopathy is the prime promoter and critical therapeutic target of the diabetic kidney disease (Gilbert, 2017). Tubulopathy is not secondary to the glomerulus, instead, it's early and original features change (Zeni et al., 2017). Moreover, the rate of renal decline was found to be more correlated with the degree of tubular damage and interstitial fibrosis (Zeni et al. 2017), thereby indicating that the renal tubular injury in the context of diabetes is hard to be ignored.

Diabetic kidney disease seems to be more complicated, as different patterns of programmed cell death may involve in the renal tubular epithelial injury (Wang et al., 2020; Qiu et al., 2016; Qin et al., 2019). Programmed cell death represents a primary approach coordinated by organisms to eliminate tubular damaged cell. The developmental programming and stress-induced signals promote this programmed cell death process, further facilitating membrane-bound and cytosolic proteins, which induce cell death by complex cascades of post-translational modifications and transcriptional alternations (Bedoui et al., 2020). Programmed cell death can be divided into apoptotic and nonapoptotic programmed cell death. Nonapoptotic programmed cell death, such as pyroptosis, necroptosis, and ferroptosis, have been revealed recently in addition to 
apoptosis (Robinson et al., 2019).

Tongxinluo is a traditional Chinese medicine containing 12 types of herbs and insects (Zhang., 2019). It exhibits valuable therapeutic potentials for heart disease $(\mathrm{Wu}$ et al., 2006), cerebral disease (Yuan et al., 2007), and kidney injury (Zhong et al., 2011). It facilitates the myocardial microvascular endothelial barrier's function by stimulating ischemia preconditioning, thereby preventing myocardial ischemia/reperfusion injury (Qi et al., 2015). Tongxinluo participates in inflammation inhibition and lipid metabolism regulation (Zhang et al., 2009; Chen et al., 2018; Chen et al., 2009) to stabilize atherosclerotic plaque (Qi et al., 2018). Tongxinluo was found to improve renal constructure and capability in diabetic kidney disease by modulating the epithelial-tomesenchymal transition and protecting podocytes from damage (Wu et al., 2016; Wang et al., 2014). However, further research is still required to investigate the effects of Tongxinluo on the kidney are related to the regulation of distinct patterns of programmed cell death in diabetic kidney disease.

\section{Materials and Methods \\ Formulation and administration}

Tongxinluo provided by Shijiazhuang Yiling Pharma-

\section{Box 1: Assessment of cell viability (MTS Assay)}

\section{Principle}

The MTS tetrazolium compound (Owen's reagent) is bioreduced by cells into a colored formazan product that is soluble in tissue culture medium. This conversion is presumably accomplished by NADPH or NADH produced by dehydrogenase enzymes in metabolically active cells. The quantity of formazan product as measured by absorbance at $490 \mathrm{~nm}$ is directly proportional to the number of living cells in culture

\section{Requirements}

MTS (3-(4, 5-dimethylthiazol-2-yl)-5-(3-carboxymethoxyphenyl)-2-(4-sulfophenyl)-2H-tetrazolium, inner salt) (CAS\#: 138169-43-4, Promega, G358B); HK-2 cells (ATCC, LGC Standards); 96-well plates; Incubator with $5 \% \mathrm{CO}_{2}$ at $37^{\circ} \mathrm{C}$; Sunrise RC microplate reader (TECAN, Switzerland); DMEM/ F12 (5 mmol/L glucose); DMEM/F12 (5 mmol/L glucose combined with $20 \mathrm{mmol} / \mathrm{L}$ mannitol); DMEM/F12 (25 mmol/ L glucose); DMEM/F12 (25 mmol/L glucose combined with $500 \mu \mathrm{mol} / \mathrm{L}$ palmitic acid); DMEM/F12 (25 mmol/L glucose, $500 \mu \mathrm{mol} / \mathrm{L}$ palmitic acid, and $400 \mu \mathrm{g} / \mathrm{mL}$ Tongxinluo)

\section{Procedure}

Step 1: The HK-2 cells were cultured in serum-free DMEM/ F12 (5 mmol/L glucose) and based on a density of $6 \times 10^{4}$ which incubated in 96-well plates for 24 hours.

Step 2: After 24 hours, when removing the media, do it carefully in order to avoid variation in data. ceutical Co., Ltd. (China) was grounded into ultrafine powder with a diameter of fewer than 10 micrometers. For the in vivo study, $0.5 \%$ sodium carboxymethylcellulose was used to dissolve the Tongxinluo ultrafine powder, and the resulting solution was given to $\mathrm{db} / \mathrm{db}$ mice daily at a dose of $0.75 \mathrm{~g} / \mathrm{kg}$ via intragastrical administration following the published procedure (Wang et al., 2014). Metformin (Glucophage, Merck Serono) dissolved in $0.5 \%$ sodium carboxymethylcellulose was used as the positive control and given to mice daily at a dose of $250 \mathrm{mg} / \mathrm{kg}$ via intragastrical administration calculated based on the maximum clinical equivalent dose (Foretz et al., 2014). For the in vitro study, serumfree Dulbecco's modified Eagle's medium/nutrient mixture F-12 (DMEM/F12) basal medium was used to dissolve the Tongxinluo ultrafine powder. And then, sonication was conducted on the solution for 1 hour, followed by centrifugation at 3,500 rpm for $10 \mathrm{~min}$. The solution was then filtrated by a micropore filter $(0.22$ $\mu \mathrm{m})$ twice. Simultaneously, the residue was heated at 60 ${ }^{\circ} \mathrm{C}$ until dryness to determine the actual volume of the Tongxinluo ultrafine powder. The final concentration of solution was adjusted to $2000 \mu \mathrm{g} / \mathrm{mL}$, and the final solution was stocked at $-20{ }^{\circ} \mathrm{C}$ until use (Wang et al., 2014).

\section{Animal experiment}

All animal protocols were established following the

Step 3: Treat the cells with a) DMEM/F12 (5 mmol/L glucose combined with $20 \mathrm{mmol} / \mathrm{L}$ mannitol, normal control); b) DMEM/F12 (25 mmol/L glucose, HG group); c) DMEM/F12 $(25 \mathrm{mmol} / \mathrm{L}$ glucose combined with $500 \mu \mathrm{mol} / \mathrm{L}$ palmitic acid, HGHP group); d) DMEM/F12 (25 mmol/L glucose, 500 $\mu \mathrm{mol} / \mathrm{L}$ palmitic acid, and $400 \mu \mathrm{g} / \mathrm{mL}$ Tongxinluo, Tongxinluo group) . The total volume in each well should be $100 \mu \mathrm{L}$.

Step 4: Cells are plated in six copies to minimize the variation in results, and incubated the cells for indicated time points (12, 24,48 , and 72 hours).

Step 5: When the incubation time point is reached, add $20 \mu \mathrm{L}$ per well of MTS.

Step 6: Incubate the plate at $37^{\circ} \mathrm{C}$ for 1.5 hours in a humidified, $5 \% \mathrm{CO}_{2}$ atmosphere.

Step 7: Record the absorbance at $490 \mathrm{~nm}$ using a Sunrise RC microplate reader.

\section{Precautions}

To measure the amount of soluble formazan produced by cellular reduction of MTS, proceed immediately to Step 7. Alternatively, to measure the absorbance at a later time, add $25 \mu \mathrm{L}$ of $10 \%$ SDS to each well to stop the reaction. Store SDStreated plates protected from light in a humidified chamber at room temperature for up to 18 hours. Proceed to Step 7.

\section{References}

Endo et al., 2018 
NIH Guide for the Care and Use of Laboratory Animals and consented by the Animal Care and Use Committee of Shijiazhuang, China. Six-week-old male $\mathrm{db} / \mathrm{db}$ mice with C57BL/Ks background and control C57BL/Ks mice were obtained from the Model Animal Research Center at Nanjing University, China. The mice were implemented as diabetic kidney disease models for the current study (Sembach et al., 2020). After two weeks of adaptation, mice were divided randomly into three groups: vehicle; Tongxinluo (0.75 mg/kg/day); metformin $(250 \mathrm{mg} / \mathrm{kg} /$ day $)$. Fifteen mice were used for each group. The Tongxinluo dose was determined according to our prior research and the literature report (Wang et al., 2014). Tongxinluo, metformin, or vehicle was started to be administrated to the 8-week-old db/ $\mathrm{db}$ mice through intragastric administration, and the treatments were maintained for 12 weeks. All mice were kept in a standardized animal facility based on a light/dark cycle of 12 hours vs. 12 hours. Bodyweight, water intake, and blood glucose levels were measured every week. 24-hour urine was collected every 4 weeks and then examined for microalbumin excretion. Random blood glucose levels were determined using a commercial glucose meter (Roche Diagnostics $\mathrm{GmbH}$, Germany). Mice were euthanized under anesthesia, and renal tissue samples were fixed in $4 \%$ paraformaldehyde overnight or stocked at $-80^{\circ} \mathrm{C}$ until analysis. At least 11 mice from each group were biologically evaluated.

\section{Cell culture}

HK-2 cells (ATCC, LGC Standards) obtained from the Procell Life Science and Technology Co., Ltd. were grown in DMEM/F12 supplemented with $100 \mathrm{U} / \mathrm{mL}$ penicillin, $10 \%$ heat-inactivated fetal bovine serum, and $100 \mu \mathrm{g} / \mathrm{mL}$ streptomycin (GIBCO, Invitrogen Corp, USA) under a humidified atmosphere consisting of $5 \%$ $\mathrm{CO}_{2}$ and $95 \% \mathrm{O}_{2}$ at $37^{\circ} \mathrm{C}$. $\mathrm{HK}-2$ cells were grown to subconfluence and rendered in serum-free medium with $5 \mathrm{mmol} / \mathrm{L}$ glucose for 24 hours prior to further treatments and then incubated with fresh $10 \%$ heatinactivated serum medium respectively containing: a) glucose $(5 \mathrm{mmol} / \mathrm{L})$ and mannitol $(20 \mathrm{mmol} / \mathrm{L})$ as the normal control group (NC group); b) glucose (25 $\mathrm{mmol} / \mathrm{L}$ ) as the high glucose group (HG group); c) glucose $(25 \mathrm{mmol} / \mathrm{L})$ and palmitic acid (PA, $500 \mu \mathrm{mol} /$ $\mathrm{L})$ as the high glucose combined with a high palmitic acid group (HGHP group); d) glucose (25 mmol/L), PA (500 $\mu \mathrm{mol} / \mathrm{L})$, and Tongxinluo $(400 \mu \mathrm{g} / \mathrm{mL})$ together as the Tongxinluo treatment group (Tongxinluo group). The HK-2 cells of the abovementioned four groups were cultured for an additional 24 hours.

\section{Histological and immunohistochemical staining}

The renal tissue samples fixed in formalin were embedded in paraffin and divided into $3 \mathrm{~mm}$ thick sections. The hematoxylin-eosin (H\&E) and periodic acid-Schiff (PAS) stainings were further performed on the sections, followed by image acquirement utilizing a light microscope. The paraffin-embedded tissues were deparaffinized for immunohistochemistry. The rehydrated sections were retrieved by microwavebased antigen quenched in $1 \% \mathrm{H}_{2} \mathrm{O}_{2}$ solution for 15 min. Furthermore, the sections were blocked using $5 \%$ rabbit serum blocking solution for 1 hour and stained for 12 hours with antibodies against collagen I (ab21286, Abcam), collagen IV (ab256353, Abcam), caspase-1 (22915-1-AP, proteintech) or IL-1 $\beta$ (ab-205924, Abcam) (dilution 1:200), respectively. Additionally, peroxidaseconjugated secondary antibodies and diaminobenzidine were applied to incubate with the sections, followed by counterstaining with hematoxylin and image acquirement by light microscope $(x 400)$.

\section{Terminal deoxynucleotidyl transferase-mediated dUTP -biotin nick end labeling assay (TUNEL) and immuno- fluorescent double staining}

The renal tissue samples were fixed in $4 \%$ paraformaldehyde dissolved in PBS for 12 hours and embedded in paraffin blocks. And then, the tissues were divided into $3 \mu \mathrm{m}$ thick sections. The TUNEL and immunofluorescence stainings were further performed on the sections. After the deparaffinization and dehydration of the tissue section, fragmentation of intracellular DNA in the kidney section was evaluated by TUNEL assay using the Fluorescein (FITC) TUNEL cell apoptosis detection kit (Servicebio, China). The nuclei stained by DAPI showed blue color under the excitation of ultraviolet light. The FITC fluorescein was applied to label the TUNEL kit. The DNA fragmentation cells were labeled with green color. The AQP1 antibody (GB11310-1, Servicebio) was labeled with red color.

\section{Western blot}

The protein lysis was performed on the HK-2 cells and renal tissues according to the manufacturer's instructions. The BCA protein standard assay kit (Beyotime, China) was utilized to determine the protein concentrations. The proteins were extracted from supernatants by StrataClean Resin (Agilent Technologies, USA) followed by separation using Bis-Tris Gel (Genscript, China) and transferred onto PVDF mem-branes (Millipore, USA) utilizing a Semi-Dry transfer system. QuickBlock $^{\mathrm{TM}}$ Blocking Buffer (Beyotime, China) was applied to block the PVDF membranes for $15 \mathrm{~min}$ at room temperature, and primary antibodies were used to incubate with these membranes overnight at $4^{\circ} \mathrm{C}$. After that, membranes were then rinsed with TBST and further incubated with secondary Alexa Fluor ${ }^{\circledR}$ antibodies for an additional 1.5 hour at room temperature. Odyssey CLx Image Studio (Odyssey, USA) was implemented to detect the signals. The relative protein expression levels normalized to $\beta$-actin were expressed as the intensity values. Anti-NF-kB p-p65 (3033, Cell Signaling Technology), anti-NLRP3 (D4D8T, Cell Signaling Technology), anti-human ASC (ab155970, 
Abcam), anti-human cleaved gasdermin D (E7H9G, Cell Signaling Technology), anti-mouse cleav-ed gasdermin D (E3E3P, Cell Signaling Technology), anticaspase-1 (22915-1-AP, proteintech), anti-human IL-1 $\beta$ (ab-33774, Abcam), anti-mouse IL-1 $\beta$ (ab-200478, Abcam), anti- $\beta$-actin (8H10D10, Cell Signaling Technology), anti-tubulin (8H10D10, ab-7291, Abcam), anticaspase-3 (ab184787, Abcam), anti-Bcl-2 (ab59348, Abcam), anti-Bax (ab32503, Abcam), anti-RIP3 (ab62344, Abcam), anti-MLKL (ab184718, Abcam), anti-GPX-4 (ab125066, Abcam), and anti-FACL4 (ACSL4) (ab155282, Abcam) antibodies were applied as the primary antibodies in this study. Anti-mouse IgG (ab216772, Abcam) and anti-rabbit IgG (ab216777, Abcam) were used as secondary Alexa Fluor ${ }^{\circledR}$ antibodies (Pyo et al, 2019, video).

\section{Statistical analysis}

Using SPSS 26.0 statistical software, first analyze the normality of each group of data, and use the method of one-way analysis of variance (One-Way ANOVA) for the data that meets the normal distribution, and then perform the homogeneity of variance test on the data. The least significant difference method is used for pairwise comparison, and the Dunnett's T3 test method is used for pairwise comparison of uneven variance. If the data does not conform to the normal distribution, non-parametric test is used for statistical analysis. All analyses were performed using GraphPad Prism 8 (GraphPad software, USA).

\section{Results}

\section{Proteinuria and renal damage in $d b / d b$ mice}

The results displayed in Figure 1A indicated that Tongxinluo reduced the urinary microalbumin excretion according to the 24-hour albuminuria excretion rate as a typical indicator of diabetic kidney disease. Furthermore, the urine albumin-creatinine ratios (UACR) were determined to measure urine protein excretion and assess the development of diabetic kidney disease. The results shown in Figure 1B further suggested that mice exhibited noticeably more generous levels of UACR relative to normal $\mathrm{C} 57 \mathrm{BL} / \mathrm{Ks}$ mice. It was noted that after four weeks of Tongxinluo administration; the levels of UACR were decreased, showing more substantial results than the positive control metformin. Concurrently, H\&E and PAS staining results also demonstrated that mice exhibited elevated vacuolation of renal tubular epithelium and slight mesangial expansion (Figure 2A). Immunostaining of collagen I and IV highlighted the development of diabetic tubulointerstitial fibrosis at week 20 of disease (Figure 2B). Tongxinluo treatment alleviated the increase in mice. Further analysis demonstrated that cell DNA fragmentation was elevated in the kidneys of mice relative to that in control mice, while these enhance-

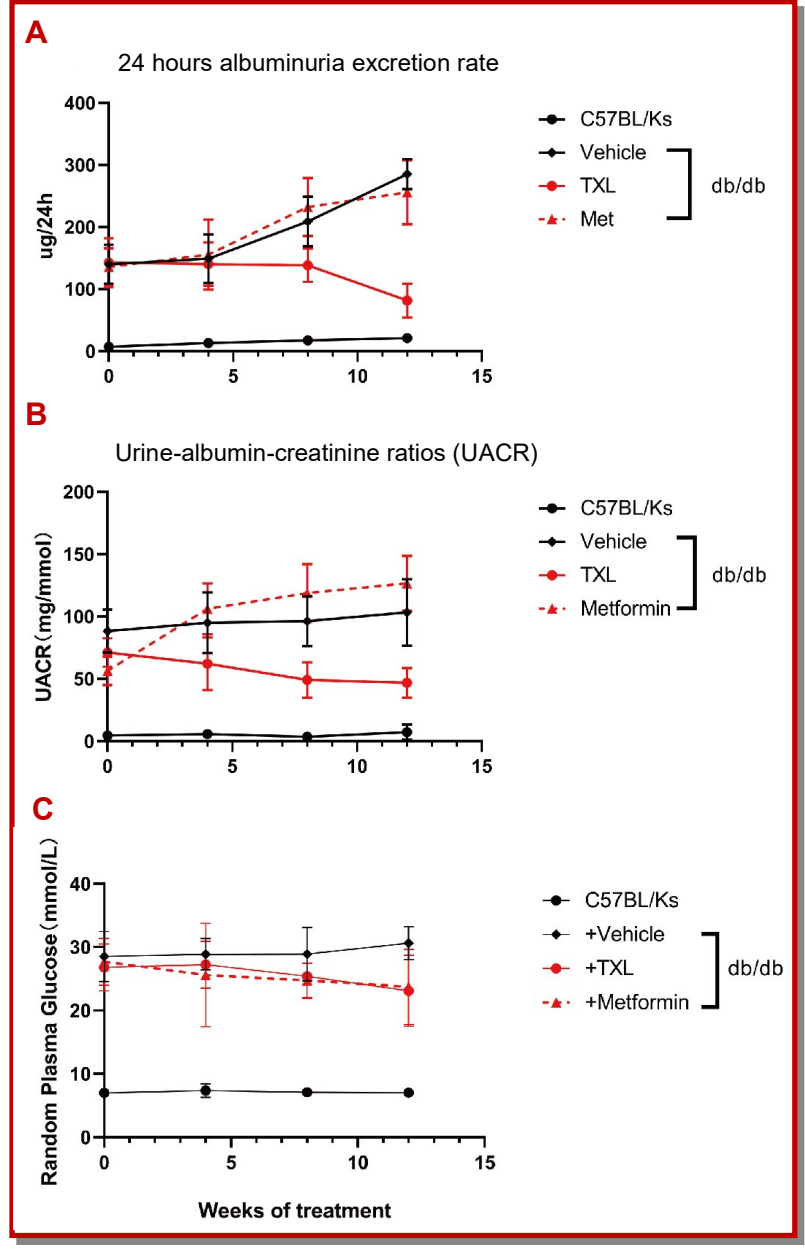

Figure 1: Effect of intervention with Tongxinluo (TXL) on albuminuria and renal function in the animal model of diabetic kidney diseases. Dynamic changes in urinary albumin excretion of mice in 24 hours every 4 weeks $(n=11)(A)$; Dynamic changes in urine protein creatinine ratio (UACR) from the start of Tongxinluo and metformin intervention to the end of the experiment $(n=11)(B)$; Random plasma glucose levels of the intervention mice as indicated were measured every 4 weeks $(\mathrm{n}=11)(\mathrm{C})$

ments were significantly attenuated by Tongxinluo administration. This improvement was more noticeable relative to metformin treatment (Figure 3).

\section{Effect on random blood glucose level}

It was found that the random plasma glucose level of $\mathrm{db} / \mathrm{db}$ mice was significantly higher than that of C57BL/Ks mice (Figure 1C). Both Tongxinluo and metformin interventions showed a mild decrease in glycemia in $\mathrm{db} / \mathrm{db}$ mice. However, no significant difference was observed in the level of RPG between the treatment groups and the vehicle mice.

\section{Protection of HK-2 cells from HGHP damage and the activation of pyroptosis and apoptosis}

MTS assessment was utilized to examine the cytotoxicity after treating $\mathrm{HK}-2$ cells with high-glucose 


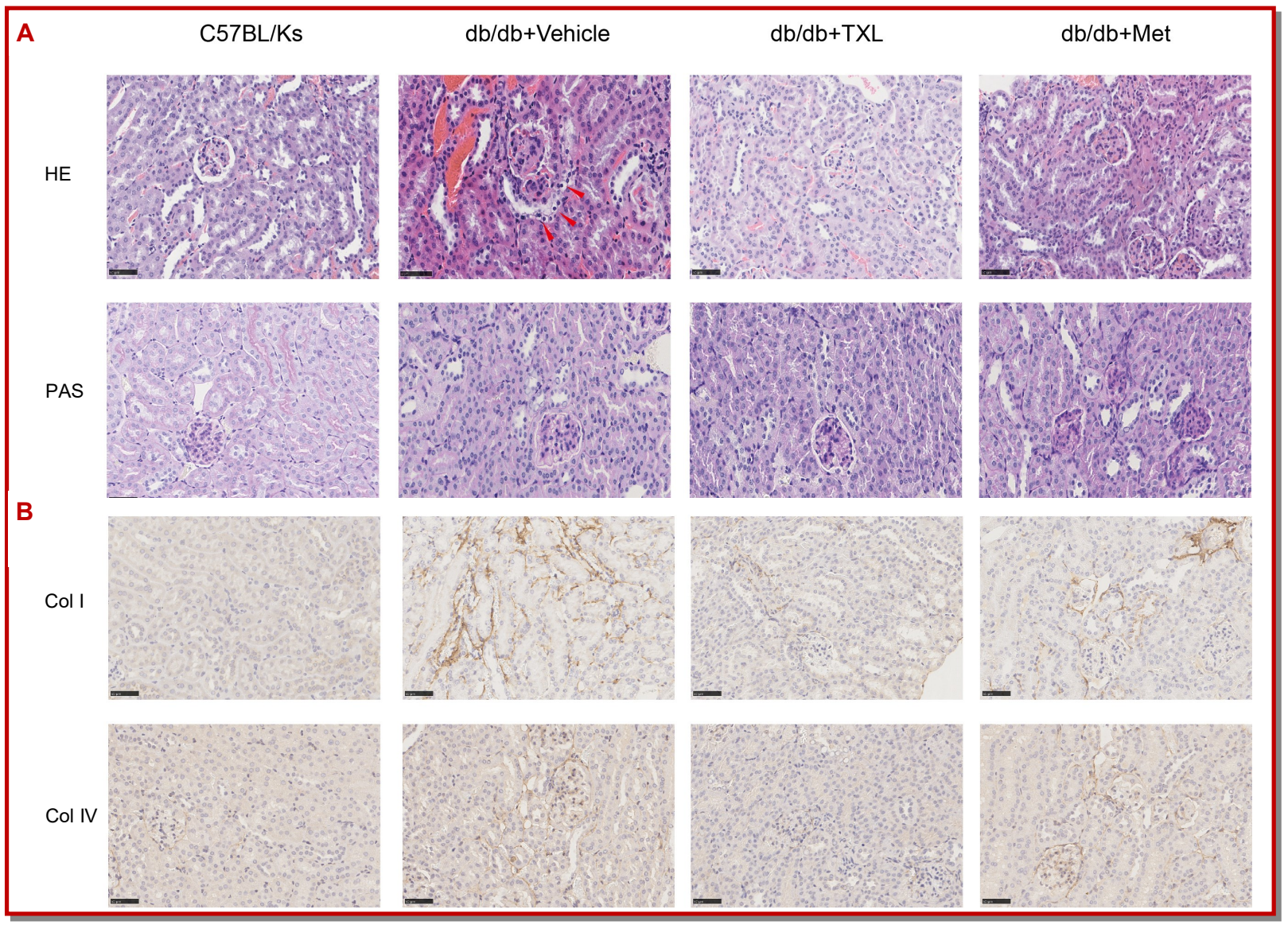

Figure 2: Tongxinluo (TXL) improves renal morphologic abnormalities and reduces interstitial collagen deposition in the kidney of $\mathrm{db} / \mathrm{db}$ mice. HE and PAS staining of kidney tissue from the investigated mice $(\times 400)(\mathrm{A})$. Scale bar, $50 \mu \mathrm{m}$; Relative col I and col IV expression levels in renal cortex from mice measured by immunohistochemical staining $(\times 400)$. Scale bar, $50 \mu \mathrm{m}(\mathrm{B})$

(HG), high-glucose combined with high-palmitic acid (HGHP), and Tongxinluo intervention. HGHP damage significantly reduced the cell viability relative to the control cells (Figure 4A). On the other hand, HG treatment increased cell viability but did not show a statistical difference compared to the control cells (Figure 4A). Moreover, Tongxinluo treatment exhibited maximum improvement in cell viability at the concentration of $400 \mathrm{~g} / \mathrm{mL}$ after 24 hours incubation (Figure $4 \mathrm{~B}$ and $4 \mathrm{C}$ ). We also found that the protein levels of NF-kB p-p65, NLRP3, ASC, caspase-1, cleaved GSDMD, and IL-1 $\beta$ were substantially down-regulated in the Tongxinluo group relative to the HGHP group (Figure 5A to 5K). NLRP3 inflammasome consisting of NLRP3, ASC, and caspase-1 results in cell lysis, death, and the release of mature IL-1 $\beta$ and IL-18, which induces pyroptosis. The activation of NF-KB is the priming step. The gasdermin family member gasdermin D (GSDMD) plays a significant role in pyroptosis, which can be divided into an $N$-terminal and a $C$ terminal fragment catalyzed by caspase-1. The released $\mathrm{N}$-terminal GSDMD fragments are relocated to the cell membrane's inner leaflet, where transmembrane pores are established, thereby building up a gap between the cytoplasm and the extracellular space. Caspase- 3 is considered as one of the important executioners in the activation of apoptosis. HGHP increased the expression of pro-caspase-3, cleaved caspase-3 and Bax and decreased the expression of $\mathrm{Bcl}-2$ and the $\mathrm{Bcl}-2 / \mathrm{Bax}$ ratio, whereas Tongxinluo treatment rescued these changes (Figure 6). However, HGHP increased the expression of necroptosis and ferroptosis-related proteins (MLKL, RIP3, GPX-4, and FACL-4), while these differences were not statistically significant (Figure 7).

\section{Suppression of the activation of pyroptosis and apoptosis in the kidney of mice}

Based on the exploration of the influence of Tongxinluo on pyroptosis and apoptosis in diabetic kidneys, we noticed that the pyroptosis was activated in the kidneys of untreated mice by up-regulating NF-kB p-p65, NLRP3, pro-caspase-1, cleaved caspase- 1 and IL-1 $\beta$, which were significantly suppressed by Tongxinluo treatment (Figure 8A to $8 \mathrm{~F}$ ). We detected comparable results in immunohistochemical staining. The immunohistochemical staining results shown in Figure 8G exhibited apparently caspase- 1 and IL- $1 \beta$ in the renal tissues of untreated mice relative to that in $\mathrm{C} 57 \mathrm{BL} / \mathrm{Ks}$ mice, which was attenuated after Tongxinluo treatment. Moreover, the apoptosis was activated in the kidneys of 


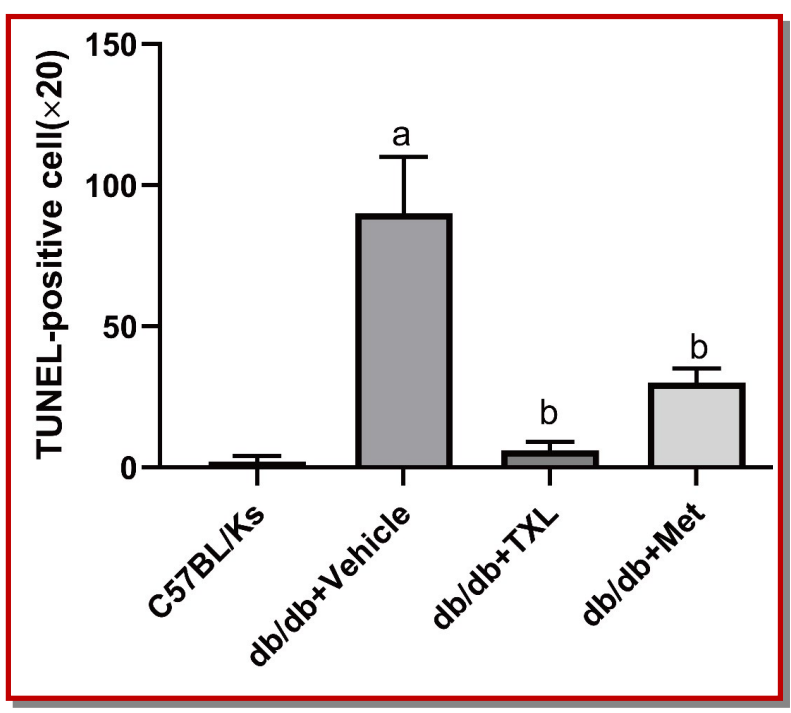

Figure 3: TUNEL-positive tubular epithelial cells in different group. ap $<0.05$ compared with C57BL/Ks control and ${ }^{b} p<0.05$ compared with undertreated $\mathrm{db} / \mathrm{db}$ mice (model group)

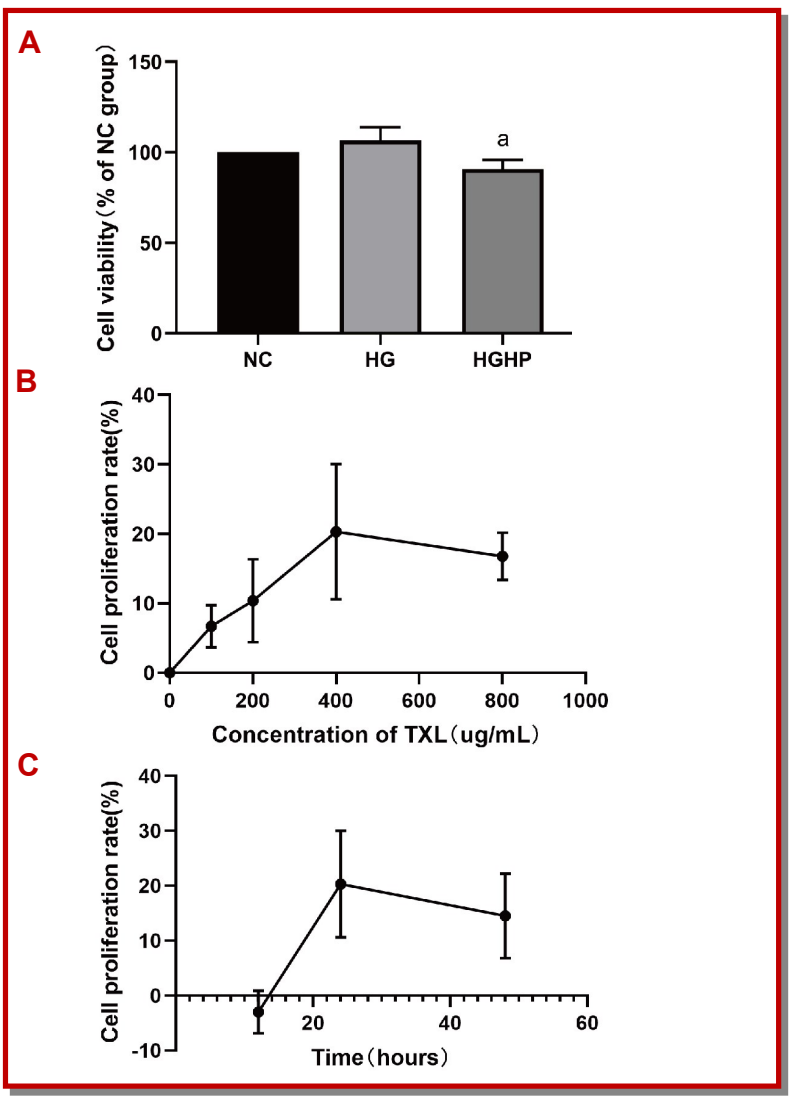

Figure 4: Tongxinluo (TXL) protects HK-2 cells from highglucose combined with high-palmitic acid (HGHP) injury. Effects of high-glucose (HG) and high-glucose combined with high-palmitic acid (HGHP) on the cell viabilities of HK-2 cells (A); Effects of different concentrations of Tongxinluo on the cell viabilities of HK-2 cells cultured in HGHP 24 hours (B); Effects of different treatment time of $400 \mu \mathrm{g} / \mathrm{mL}$ Tongxinluo on the cell viabilities of HK-2 cells cultured in HGHP (C) . NC means normal control mice by upregulated levels of caspase- 3 and Bax and decreased the Bcl-2/Bax ratio, which were reversed by Tongxinluo treatment (Figure 9).

\section{Discussion}

In the current work, we found that Tongxinluo protected renal function by improving renal morphologic abnormalities, reducing urinary albumin excretion in the diabetic kidney disease model of $\mathrm{db} / \mathrm{db}$ mouse.

To validate the rodent model of diabetic kidney disease, 12 weeks long-term feeding in mice was reproduced according to the previous study (Tesch et al., 2011). The albuminuria excretion rate were more than 10 times in $\mathrm{db} / \mathrm{db}$ mice than that of C57BL/Ks mice before the administration. After 12 weeks of treatment, the renal histological abnormalities, including vacuolation of renal tubular epithelium and slight mesangial expansion, were improved noticed in $\mathrm{db} / \mathrm{db}$ mice. And no apparent inflammatory infiltrates and tubular necrosis were observed. Tongxinluo also attenuated the levels of collagen I and IV in the $\mathrm{db} / \mathrm{db}$ mice's kidneys. These results indicated that this mouse model were more in line with the early stage of diabetic kidney disease.

It was found that Tongxinluo prevented renal injury in diabetic kidney disease by decreasing the levels of AER and UACR and improving renal morphologic abnormalities. In comparison with metformin, Tongxinluo showed a better protective effect. Although it is valuable for the use of metformin in the treatment of diabetic kidney disease, we found that metformin had no significant effect on lowering RPG in this study. Similarly, metformin did not alter fasting glucose level and body weight gain (Mitchell et al., 2017). Furthermore, they speculated that this might be related to the mouse model (BKS db), which has more pronounced insulin resistance compared to other $\mathrm{db} / \mathrm{db}$ mouse models, causing decreased lipogenesis (Davis et al., 2010). A prior study demonstrating that metformin is mainly effective in the liver and does not apparently promote peripheral insulin sensitivity (Natali and Ferrannini, 2006). We also found the renal tubular epithelium cells in mice showed substantial fragmentation of their DNA by TUNEL assay which was inhibited after Tongxinluo treatment.

The tubular injury caused by hyperglycemia is associated with various mechanisms, such as increased expression of the extracellular matrix, production of advanced glycation products, and activity of the Wnt/ $\beta$ catenin pathway and protein kinase $C$ (Kanwar et al., 2011). A high level of glucose has been widely implemented to trigger stress in most experimental 


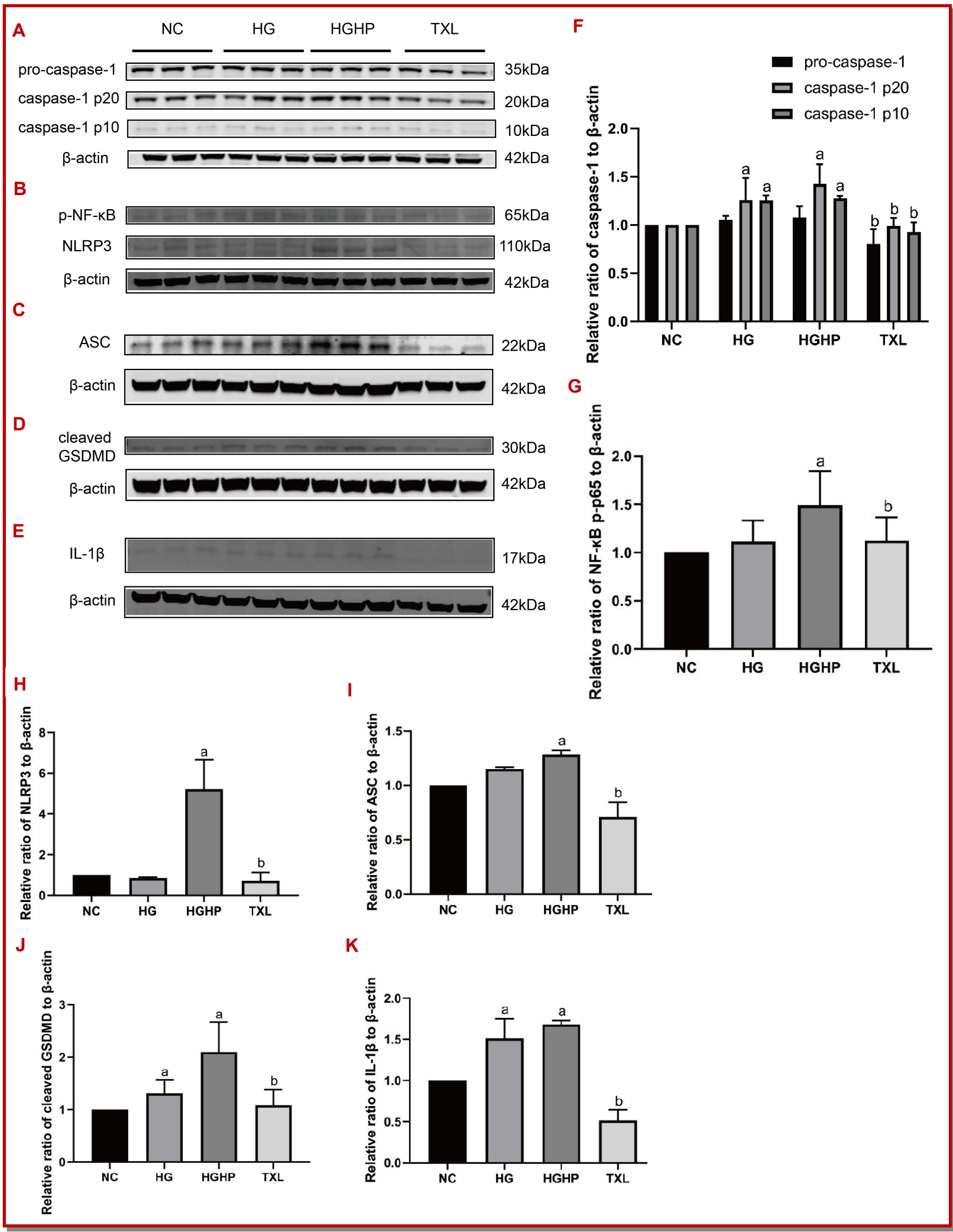

Figure 5: Effect of Tongxinluo (TXL) on pyroptosis in HK-2 cells exposed to high-glucose (HG) and high-glucose combined with high-palmitic acid (HGHP). (A E) Representative band of NF-kB p-p65, NLRP3, ASC, Caspase-1, cleaved GSDMD and IL-1 $\beta$ by Western blot in vitro $(\mathrm{n}=3$ per group). ( $\mathrm{F} \sim \mathrm{K})$ Semiquantitative analysis of average optical density of NF-kB p-p65, NLRP3, ASC, Caspase-1, cleaved GSDMD and IL-1 $\beta$. The values represent means \pm SEM. ${ }^{a} p<0.05$ : the significant difference with respect to normal control (NC) group; b $<<0.05$ : the significant difference with respect to HGHP group 


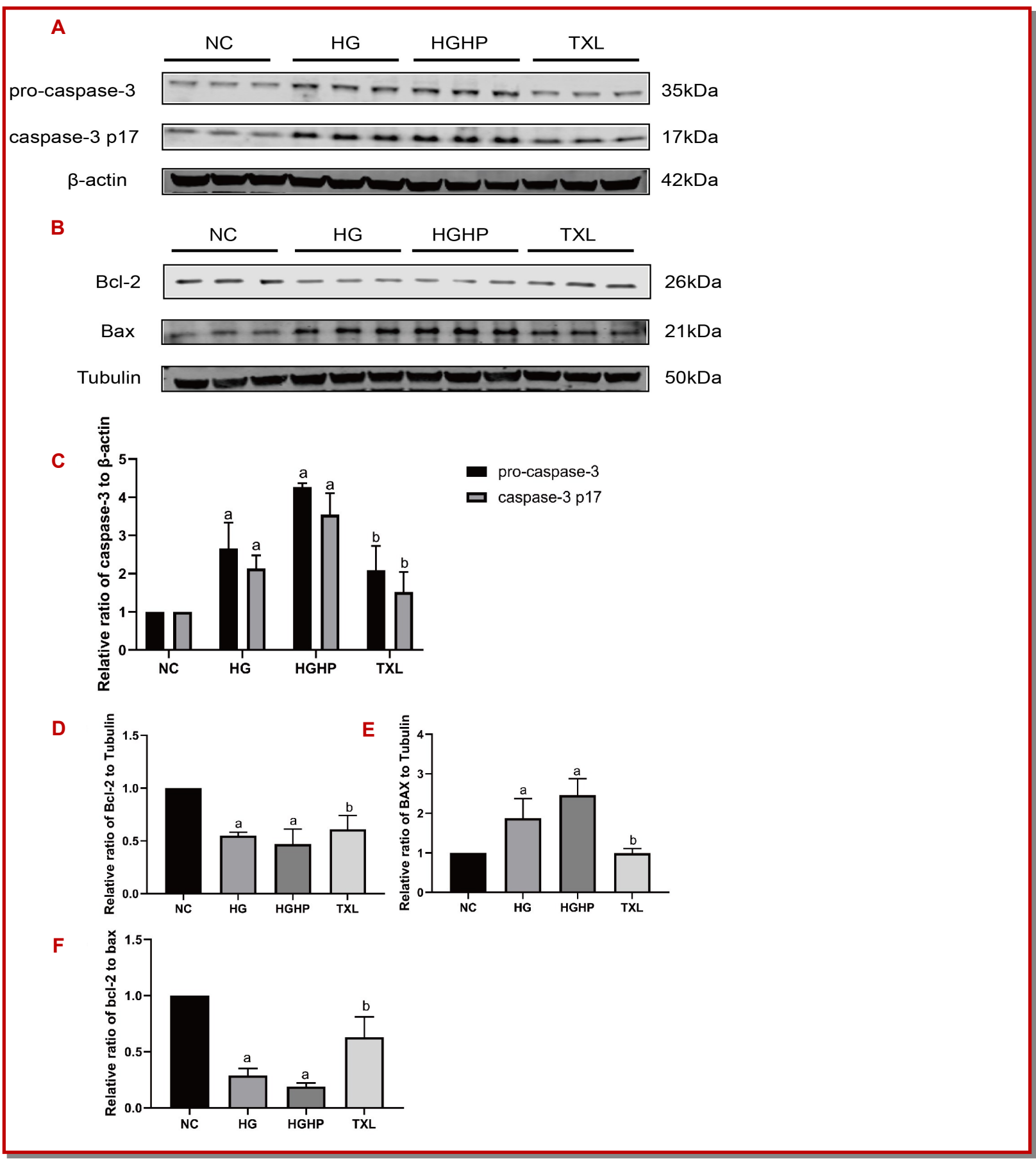

Figure 6: Effect of Tongxinluo (TXL) on apoptosis in the HK-2 cells exposed to high-glucose (HG) and high-glucose combined with high-palmitic acid (HGHP). Representative band of caspase-3, Bcl-2 and Bax by Western blot in vitro ( $\mathrm{n}=3$ per group) (A, B); Semiquantitative analysis of average optical density of caspase-3, Bcl-2 and Bax (C E); Relative ratio of Bcl-2 to Bax (F). The values represent means \pm SEM. ap $<0.05$ : the significant difference with respect to normal control $(\mathrm{NC})$ group; ${ }^{b} p<0.05$ : the significant difference with respect to HGHP group

studies of diabetic kidney disease. In contrast, clinical data have demonstrated that some patients with poor glycemic control do not develop nephropathy. On the contrary, renal complications

occasionally occur even if glucose control is accompli- shed. Considering that glucose and lipid metabolism is inseparable, and in addition to high glucose injury, lipotoxicity is generally seemed like one of the primary diabetic kidney disease's pathogenic factors, resulting in oxidative stress damage and enhancing inflammation. It was evident by early-stage studies that extensive 


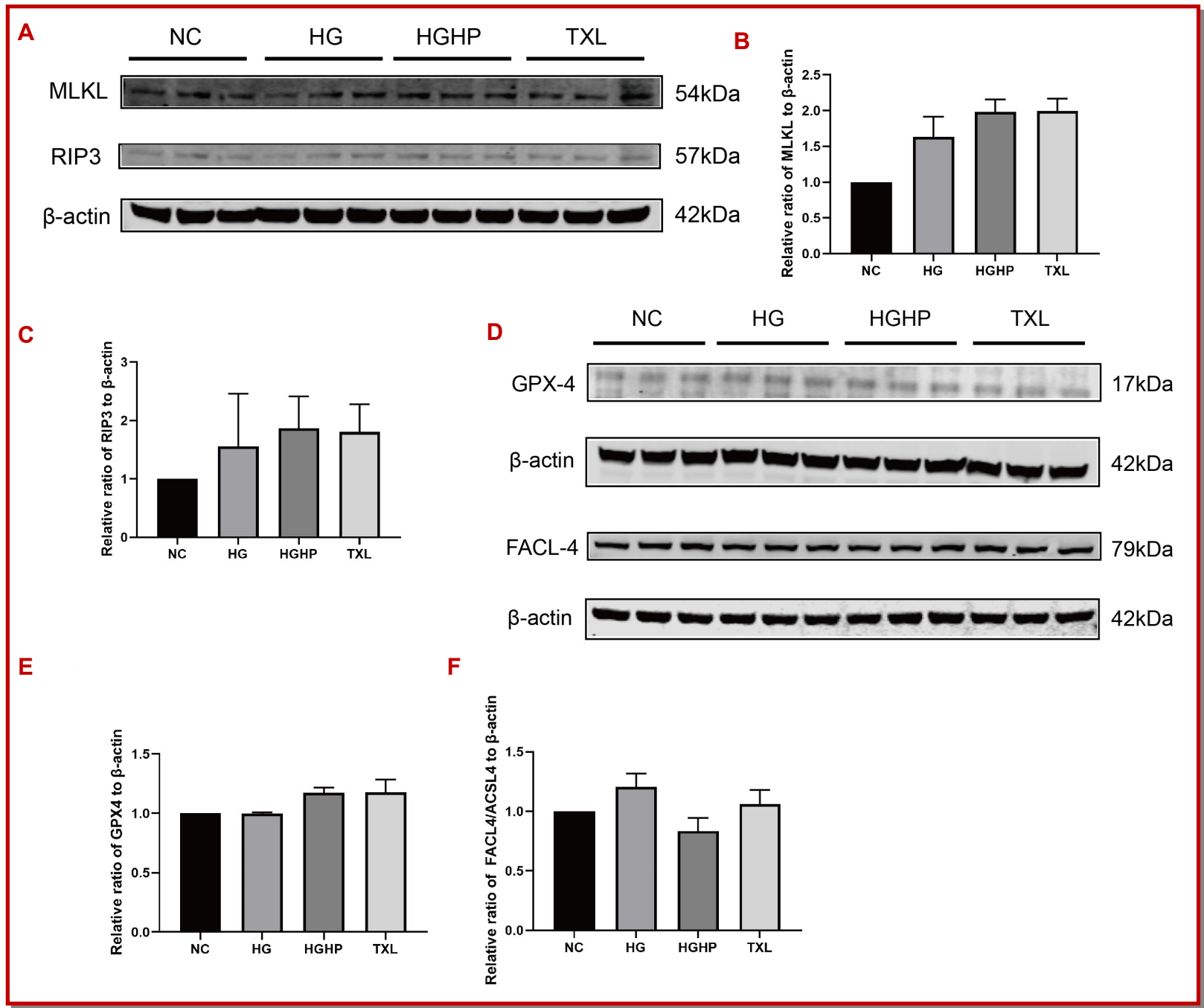

Figure 7: Effects of Tongxinluo (TXL) on necroptosis and ferroptosis in the HK-2 cells exposed to high-glucose (HG) and highglucose combined with high-palmitic acid (HGHP). Representative band of MLKL, RIP3, GPX-4 and FACL-4 (ACSL-4) by Western blot in vitro ( $\mathrm{n}=3$ per group) (A, D); Semiquantitative analysis of average optical density of MLKL, RIP3, GPX-4 and FACL-4 $(\mathrm{ACSL}-4)(B, C, E, F)$. NC means normal control. The values represent means $\pm \mathrm{SEM}$

free fatty acids and lipid accumulation are responsible for the dysfunction of mitochondria and induces apoptosis in kidney disorders (Hua et al., 2015). In vitro, we compared the cell viability of HK-2 cultured by HG and HGHP. The results showed that HG alone stimulated cell proliferation, although there was no statistical difference compared to the NC group. The significantly decreased cell viability observed in the HGHP group was prevented by Tongxinluo treatment.

According to the in vivo result of TUNEL and the in vitro effect of HGHP on cell viability, we speculated that PCD in renal tubular epithelial cells could be induced under hyperglycemia combined with hyperlipidemia.

Pyroptosis induced by NLRP3 inflammasome participates in the pathogenesis of various renal disorders, such as diabetic kidney disease (Moreno et al., 2018). Moreover, it is also suggested that pyroptosis is strongly related to renal tubular epithelial-mesenchymal transition, promoted by TGF- $\beta 1$ signaling enhancement (Wang et al., 2013). Another study found that ASC -knockout mice were protected against proteinuria and diabetic kidney disease development (Hutton et al., 2016). Our data showed that the levels of pyroptosisrelated proteins, including NF-кB p-p65, NLRP3, ASC, caspase- 1 , cleaved GSDMD and IL- $1 \beta$ were increased in the HGHP group that was reduced after treating with Tongxinluo. Similar to pyroptosis, necroptosis also belongs to inflammatory types of PCD that require the membrane damaging GSDMD and MLKL (Frank and Vince, 2018). Although necroptois-related proteins MLKL and RIP3 were upregulated in the HG and HGHP groups, there was no statistical difference compared to the NC group. Now-a-days, ferroptosis is considered to be a primary cause of cell death related to a variety of disorders, including cancers, diabetes, neurodegenerative disorders, as well as renal failure 


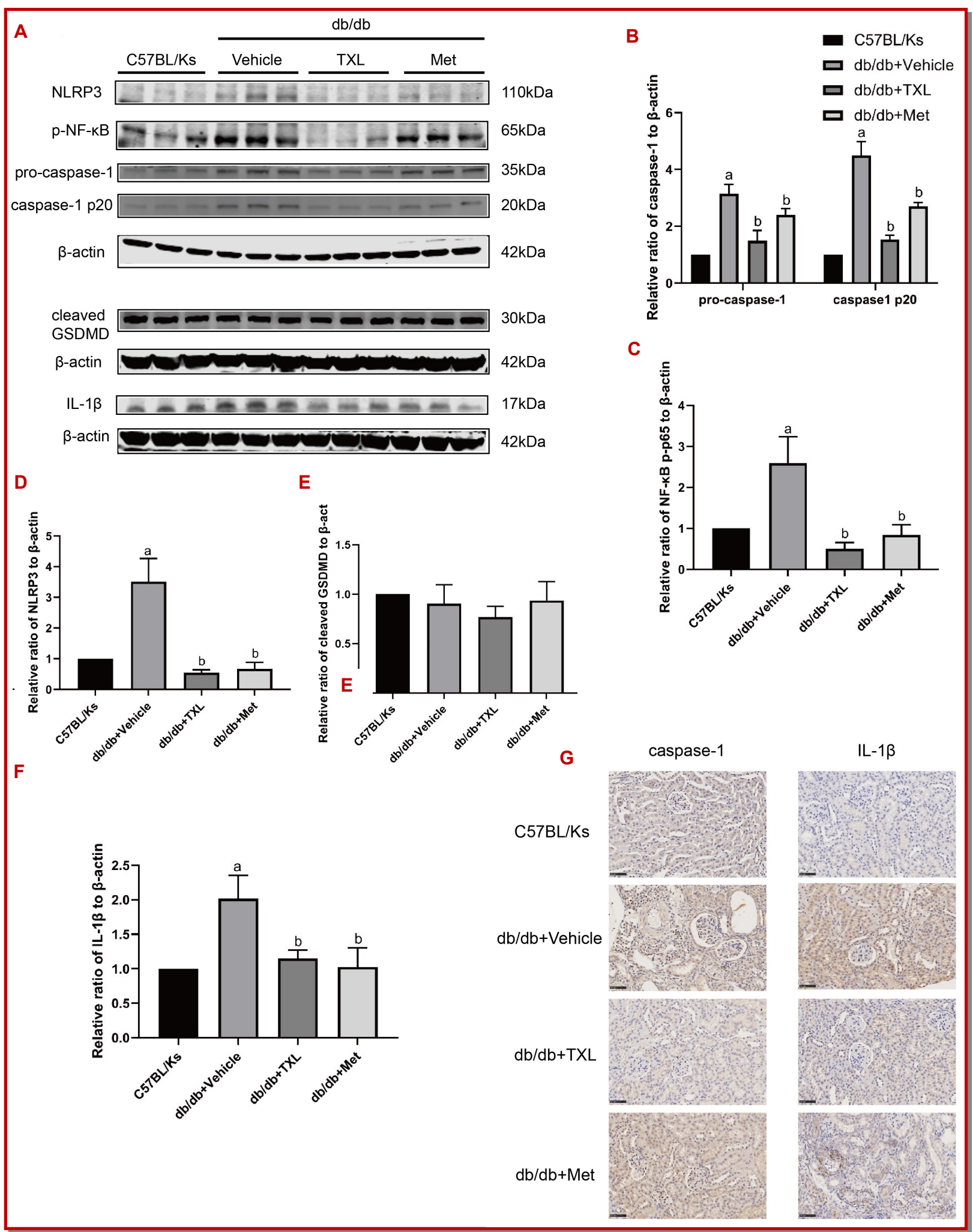

Figure 8: Effects of Tongxinluo (TXL) and metformin on pyroptosis in the kidney of $\mathrm{db} / \mathrm{db}$ mice. Representative band of NF-kB pp65, NLRP3, caspase-1, cleaved GSDMD and IL-1 $\beta$ by Western blot in the kidneys in the different groups of mice $(n=3$ per group) (A); Semiquantitative analysis of average optical density of NLRP3, p-NFkB, Caspase-1, cleaved GSDMD and IL-1 $\beta$ (B F). The values represent means \pm SEM. ap $<0.05$ : the significant difference with respect to C57BL/Ks control; b $<<0.05$ : the significant difference with respect to undertreated $\mathrm{db} / \mathrm{db}$ mice (model group). Immunohistochemical staining was performed to analyze the expression of caspase-1 and IL-1 $\beta(\times 400)$ in renal cortex from mice. Scale bar, $50 \mu \mathrm{m}(\mathrm{G})$ 


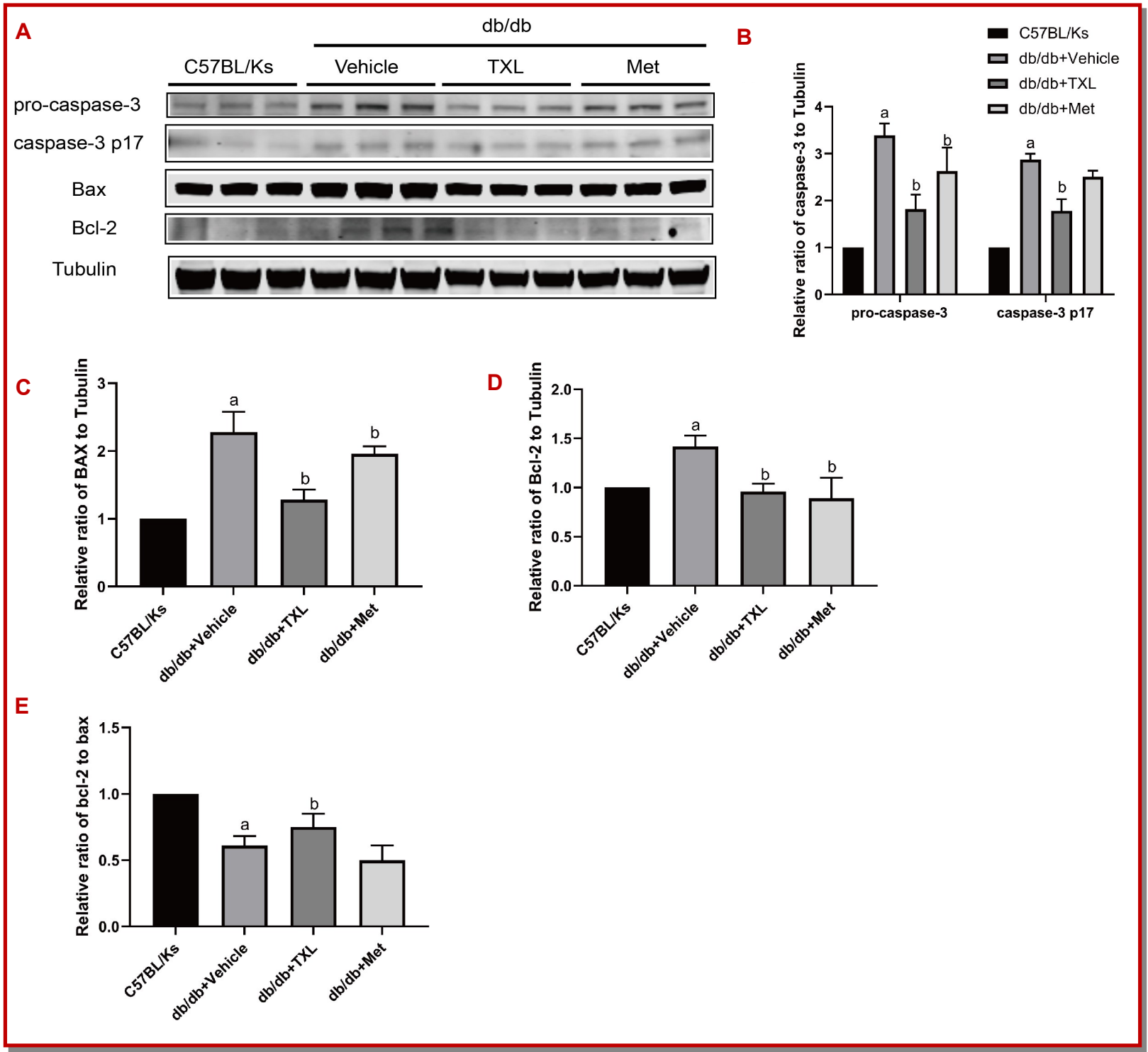

Figure 9: Effects of Tongxinluo (TXL) and metformin on apoptosis in the kidney of $\mathrm{db} / \mathrm{db}$ mice. Representative band of caspase-3, Bax, Bcl-2 by Western blot in the kidneys in the different groups of mice ( $\mathrm{n}=3$ per group) (A); Semiquantitative analysis of average optical density of caspase-3, Bax and Bcl-2 (B D ). Relative ratio of Bcl-2 to Bax (E). ap<0.05: the significant difference with respect to $\mathrm{C} 57 \mathrm{BL} / \mathrm{Ks}$ control; ${ }^{\mathrm{b}} \mathrm{p}<0.05$ : the significant difference with respect to undertreated $\mathrm{db} / \mathrm{db}$ mice (model group)

(Stockwell et al., 2017). A study showed that ferroptosis was activated in the streptozotocin-induced DBA/2J diabetic mice and HG cultured HK-2 cells (Li et al., 2021). ACSL4 (FACL4), the biomarker of ferroptosis, was not upregulated, and the expression of GPX-4 was not obviously altered in the HGHP group. Furthermore, the expression of the pyroptosis-related protein, including NF- $\mathrm{kB}$ p-p65 and ASC, in the HG group was increased and the expression of NLRP3 was decreased. At the same time, no statistical difference was observed between the HG group and the NC group. Perhaps lipotoxicity in diabetic kidney disease should be paid more attention than glucotoxicity.

The apoptosis of renal tubular epithelial cells in the hypoxic milieu in diabetic kidney disease is a signifi- cant factor that causes renal functional and pathological changes (Leonard et al., 2003; Kim et al., 2011). Our data showed that the caspase- 3 activation by HGHP could be reversed by Tongxinluo treatment. Moreover, we observed that Tongxinluo inhibited the Bax (proapoptotic effectors) expression, enhanced the Bcl-2 (anti -apoptotic proteins) expression and increased the Bcl2/Bax ratio in HK-2 under HGHP. Our data in vitro validated that Tongxinluo inhibited the activation of apoptosis and pyroptosis in HK-2 cells induced by HGHP.

We further detected the expression of apoptosis and pyroptosis related proteins of mice kidney. The expression of NF-kB p-p65, all components of the NLRP3 inflammasome, caspase- 3 and Bax was enhanced and 
the ratio of $\mathrm{Bcl}-2 / \mathrm{Bax}$ was decreased in the kidney of untreated $\mathrm{db} / \mathrm{db}$ mice. Tongxinluo treatment reversed the expression of these proteins.

\section{Conclusion}

The study shows that a) hyperglycemia combined with hyperlipidemia may be responsible for renal tubular injury; b) Tongxinluo reduces urinary albumin excretion in $\mathrm{db} / \mathrm{db}$ mice; $\mathrm{c}$ ) the nephroprotective efficacy of Tongxinluo may be partially attributed to the apoptosis inhibition and pyroptosis inhibition of the renal tubular cells.

\section{Financial Support}

National key research and development plan 'Chinese medicine modernization research' key special funding project (No. 2017YFC1700500)

\section{Ethical Issue}

All animal protocols were established following the NIH Guide for the Care and Use of Laboratory Animals and consented by the Animal Care and Use Committee of Shijiazhuang, China. The usage of drugs was in line with the Organization for Economic Co-operation and Development (OECD) guidelines for the Testing of Chemicals. Great attempts were made to minimize the number and suffering of animals. All mice were kept in a standardized animal facility based on a light/dark cycle of $12 \mathrm{~h}$ vs. $12 \mathrm{~h}$, and they were given sufficient water, food and nutrient substances.

\section{Conflict of Interest}

Authors declare no conflict of interest

\section{Acknowledgement}

All the author of this manuscript is thankful to National Key Laboratory of Collateral Disease Research and Innovative Chinese Medicine, Shijiazhuang 050011, China for providing the laboratory facility for the conduction of experiments.

\section{References}

Anders HJ, Huber TB, Isermann B, Schiffer M. CKD in diabetes: Diabetic kidney disease versus nondiabetic kidney disease. Nat Rev Nephrol. 2018; 14: 361-77.

Bedoui S, Herold MJ, Strasser A. Emerging connectivity of programmed cell death pathways and its physiological implications. Nat Rev Mol Cell Biol. 2020; 21: 678-95.

Chen WQ, Zhong L, Zhang L, Ji XP, Zhao YX, Zhang C, Jiang $\mathrm{H}$, Wu YL, Zhang Y. Chinese medicine tongxinluo significantly lowers serum lipid levels and stabilizes vulnerable plaques in a rabbit model. J Ethnopharmacol. 2009; 124: 10310.

Chen Y, Li M, Zhang Y, Di M, Chen W, Liu X, Yu F, Wang H, Zhen $X$, Zhang M. Traditional Chinese medication Tongxinluo attenuates apoptosis in ox-LDL-stimulated macrophages by enhancing Beclin-1-induced autophagy. Biochem Biophys Res Commun. 2018; 501: 336-42.

Frank D, Vince JE. Pyroptosis versus necroptosis: Similarities, differences, and crosstalk. Cell Death Differ. 2018; 26: 99-114.

Davis RC, Castellani LW, Hosseini M, Ben-Zeev O, Mao HZ, Weinstein MM, Jung DY, Jun JY, Kim JK, Lusis AJ, Péterfy M. Early hepatic insulin resistance precedes the onset of diabetes in obese C57BLKS-db/db mice. Diabetes 2010; 59: 1616-25.

Endo H, Owada S, Inagaki Y, Shida Y, Tatemichi M. Glucose starvation induces LKB1-AMPK-mediated MMP-9 expression in cancer cells. Sci Rep. 2018, 8: 10122.

Epstein FH, Remuzzi G, Bertani T. Pathophysiology of progressive nephropathies. N Engl J Med. 1998; 339: 1448-56.

Foretz M, Guigas B, Bertrand L, Pollak M, Viollet B. Metformin: From mechanisms of action to therapies. Cell Metab. 2014; 20: 953-66.

Gilbert RE. Proximal tubulopathy: Prime mover and key therapeutic target in diabetic kidney disease. Diabetes 2017; 66: 791-800.

Hua W, Huang HZ, Tan LT, Wan JM, Gui HB, Zhao L, Ruan XZ, Chen XM, Du XG. CD36 mediated fatty acid-induced podocyte apoptosis via oxidative stress. PloS one. 2015; 10: e0127507.

Hutton HL, Ooi JD, Holdsworth SR, Kitching AR. The NLRP3 inflammasome in kidney disease and autoimmunity. Nephrology 2016; 21: 736-44.

Kanwar YS, Sun L, Xie P, Liu FY, Chen S. A glimpse of various pathogenetic mechanisms of diabetic nephropathy. Annu Rev Pathol. 2011; 6: 395-423.

Kim YJ, Kim YA, Yokozawa T. Pycnogenol modulates apoptosis by suppressing oxidative stress and inflammation in high glucose-treated renal tubular cells. Food Chem Toxicol. 2011; 49: 2196-201.

Leonard MO, Cottell DC, Godson C, Brady HR, Taylor CT. The role of HIF-1 $\alpha$ in transcriptional regulation of the proximal tubular epithelial cell response to hypoxia. J Biol Chem. 2003; 278: 40296-304.

Li S, Zheng L, Zhang J, Liu X, Wu Z. Inhibition of ferroptosis by up-regulating Nrf2 delayed the progression of diabetic nephropathy. Free Radic Biol Med. 2021; 162: 435-49.

Mitchell PL, Nachbar R, Lachance D, St-Pierre P, Trottier J, Barbier $\mathrm{O}$, et al. Treatment with a novel agent combining docosahexaenoate and metformin increases protectin DX and IL-6 production in skeletal muscle and reduces insulin resistance in obese diabetic $\mathrm{db} / \mathrm{db}$ mice. Diabetes Metab. 2017; 19: 313-19.

Moreno JA, Gomez-Guerrero C, Mas S, Sanz AB, Lorenzo O, Ruiz-Ortega M, Opazo L, Mezzano S, Egido J. Targeting inflammation in diabetic nephropathy: A tale of hope. Expert Opin Investig Drugs. 2018; 27: 917-30. 
Natali A, Ferrannini E. Effects of metformin and thiazolidinediones on suppression of hepatic glucose production and stimulation of glucose uptake in type 2 diabetes: A systematic review. Diabetologia 2006; 49: 434-41.

Pyo JS, Kim HH, Kim KM, Kang JS. Amelioration of dry eye syndrome by oral administration of cultivated wild ginseng extract. Bangladesh J Pharmacol. 2019; 14: 61-66.

Qi K, Li L, Li X, Zhao J, Wang Y, You S, Hu F, Zhang H, Cheng Y, Kang S, Cui H. Cardiac microvascular barrier function mediates the protection of Tongxinluo against myocardial ischemia/reperfusion injury. PloS one. 2015; 10: e0119846.

Qi K, Li X, Geng Y, Cui H, Jin C, Wang P, Li Y, Yang Y. Tongxinluo attenuates reperfusion injury in diabetic hearts by angiopoietin-like 4-mediated protection of endothelial barrier integrity via PPAR-a pathway. PloS One. 2018; 13: e0198403.

Qin G, Huang F, Wang Q, Guo F, He Y. FoxO1-mediated inhibition of STAT1 alleviates tubulointerstitial fibrosis and tubule apoptosis in diabetic kidney disease. SSRN Electronic J. 2019; 48: 491-504.

Qiu YY, Tang LQ. Roles of the NLRP3 inflammasome in the pathogenesis of diabetic nephropathy. Pharm Res. 2016: 25164.

Robinson N, Ganesan R, Hegeds C, Kovács K, Virág L. Programmed necrotic cell death of macrophages: Focus on pyroptosis, necroptosis, and parthanatos. Redox Biol. 2019; 26: 2213-317.

Sembach FE, Østergaard MV, Vrang N, Feldt-Rasmussen B, Fosgerau K, Jelsing J, Fink LN. Rodent models of diabetic kidney disease: Human translatability and preclinical validity. Drug Discov Today. 2020; 2020.

Stockwell BR, Angeli JPF, Bayir H. Ferroptosis: A regulated cell death nexus linking metabolism. Redox Biol Dis Cell. 2017; 171: 273-85.

Tesch GH, Lim AKH. Recent insights into diabetic renal injury from the $\mathrm{db} / \mathrm{db}$ mouse model of type 2 diabetic nephropathy. Am J Physiol Renal Physiol. 2011; 300: 301-10.

Wang JY, Gao YB, Zhang N, Zou DW, Xu LP, Zhu ZY, Li JY, Zhou SN, Cui FQ, Zeng XJ, Geng JG. Tongxinluo amelio- rates renal structure and function by regulating miR-21induced epithelial-to-mesenchymal transition in diabetic nephropathy. Am J Physiol. Renal Physiol. 2014; 306: F48695.

Wang W, Wang X, Chun J, Vilaysane A, Clark S, French G, Bracey NA, Trpkov K, Bonni S, Duff HJ, Beck PL. Inflammasome-independent NLRP3 augments TGF- $\beta$ signaling in kidney epithelium. J Immunol. 2013; 190: 1239-49.

Wang Y, Bi R, Quan F, Cao Q, Zhang D. Ferroptosis involves in renal tubular cell death in diabetic nephropathy. Eur J Pharmacol. 2020; 888: 173574.

Wu T, Harrison RA, Chen XY, Ni J, Zhou L, Qiao J, Wang Q, Wei J, Duan $X$, Zheng J. Tongxinluo (Tong xin luo or Tongxin-luo) capsule for unstable angina pectoris. Cochrane Database Syst Rev. 2006.

Wu XM, Gao YB, Cui FQ, Zhang N. Exosomes from high glucose-treated glomerular endothelial cells activate mesangial cells to promote renal fibrosis. Biol Open. 2016; 5: 48491.

Yuan GQ, Wu YL, Jia ZH. Experimental study on effect of Tongxinluo on nerve cell apoptosis after cerebral ischemia in middle cerebral arterial obstructive model rats. Zhongguo Zhong Xi Yi Jie He Za Zhi. 2007; 27: 720-23.

Zeni L, Norden AGW, Cancarini G, Unwin RJ. A more tubulocentric view of diabetic kidney disease. J Nephrol. 2017; 30: 701-17.

Zhang L, Liu Y, Lu XT, Wu YL, Zhang C, Ji XP, Wang R, Liu $\mathrm{CX}$, Feng JB, Jiang $\mathrm{H}, \mathrm{Xu} X \mathrm{X}$. Traditional Chinese medication Tongxinluo dose-dependently enhances stability of vulnerable plaques: A comparison with a high-dose simvastatin therapy. Am J Physiol Heart C. 2009; 297: H2004-14.

Zhang M, Liu Y, Xu M, Zhang L, Liu X, Zhao Y, Zhu F, Xu R, Ou Z, Wang Y, Liu $Q$. Carotid artery plaque intervention with Tongxinluo capsule (CAPITAL): A multicenter randomized double-blind parallel-group placebo-controlled study. Sci Rep. 2019; 9: 4545.

Zhong X, Chung AC, Chen HY, Meng XM, Lan HY. Smad3mediated upregulation of miR-21 promotes renal fibrosis. J Am Soc Nephrol. 2011; 22: 1668-81. 